\title{
Application of 3D wavelet transforms for crack detection in rotor systems
}

\author{
C NAGARAJU ${ }^{\mathrm{a} *}, \mathrm{~K}$ NARAYANA RAO $^{\mathrm{b}}$ and \\ K MALLIKARJUNA RAO ${ }^{\mathrm{c}}$
}

${ }^{a}$ Mechanical Engineering Department, V R Siddhartha Engineering College, Vijayawada 520007

${ }^{b}$ All India Council for Technical Education (AICTE), New Delhi 110001

${ }^{\mathrm{c}}$ Mechanical Engineering Department, J N T U College of Engineering,

Kakinada 110002

*e-mail: nagaraju_cherukuri@yahoo.co.in

MS received 15 December 2007; revised 5 May 2009

\begin{abstract}
The dynamics and diagnostics of a cracked rotor have been gaining importance in recent years. The early detection of faults like fatigue cracks in rotor shafts are very important to prevent catastrophic failure of the rotor system. Vibration monitoring during start up or shut-down is as important as during steady state operation to detect cracks especially for machines such as aircraft engines which start and stop quite frequently and run at high speeds. So, the transient data of the cracked rotor has been transformed using the wavelet transforms for crack detection. Most of the works quoted in the literature used 1D wavelets or 2D wavelets (Continuous Wavelet Transform-CWT) for crack detection. The crack detectors in the signals are both time as well as frequency dependent. So, the use of $2 \mathrm{D}$ wavelets is also not enough to detect the crack. In the present work a 3D wavelet (CWT) has been utilized which clearly indicates both the time and frequency features of the crack. The presence of sub-criticals in the CWT may be a best crack indicator but it is not always reliable. The addition of noise to the signal may sometimes lead to inaccurate results. So, there is a need to identify a parameter in addition to the sub-criticals. The phase angle between the two signals (cracked and un-cracked) or two transverse vibrations can be a better crack indicator because it is very less sensitive to noise disturbance. So, to extract the above phase angle a new transform has been applied called Cross Wavelet Transform (XWT). The XWT is exploited for the first time to a rotor fault detection system in the present work. Some interesting results have been obtained using the same. The advantage of the XWT is that both, the phase angles between the transverse signals and also the amplitudes of sub-criticals are viewed in a single plot. Parametric analysis is also carried out by varying crack depth and crack position for diagnostic purposes. The inverse problem of crack identification (i.e. determining the crack parameters through known vibration data) has also been carried out using Artificial Neural Network (ANN).
\end{abstract}


Keywords. Cracked rotor; CWT; cross wavelet transform (XWT); diagnostics; shaft crack.

\section{Introduction}

In large rotating machinery, such as turbine, generator and aero-engine, the rotor is one of the most important parts. Appearances and expansion of fatigue crack in large rotating machines may lead to catastrophic failures. Online detection of cracks is very important for engineers working in the areas of machine dynamics. The research in the past few decades on cracked structures and rotors is well documented in review papers (Wauer 1990; Dimarogonas 1996; Sabnavis et al 2004). Vibration monitoring during start-up or shut-down is as important as during steady-state operations to detect cracks especially for machines such as aircraft engines which start and stop quite frequently and run at high speeds. To capture the run-up data of the rotor system the transient analysis has been applied. The importance of the transient data in detecting cracks has been well documented (Sekhar \& Prabhu 1994; Prabhakar et al 2001). To extract the hidden features of the crack the time signal obtained from the transient analysis has to be processed or transformed to different domains like FFT, wavelet transform, etc. Depending on the characteristics of the signal like linear or non-linear, stationary or nonstationary the signal processing technique has to be selected. The use of wavelet transform has proven to be better tool for crack detection (Prabhakar et al 2001), the appearance of subcritical peaks is an indication of crack in the system. Most of the early works (Prabhakar et al 2001; Sekhar 2003) used either 1D or 2D CWT's for crack detection. The crack indicators are both time as well as frequency dependent. The use of 2D CWT (which is time vs. amplitude) may detect the frequency component but it cannot indicate the frequency component quantitatively.

So, to overcome the above problem a new wavelet plot called 3D CWT has been applied which clearly shows the time-frequency-amplitude. Using the above $3 \mathrm{D}$ plots the crack features can be quantitatively as well as qualitatively detected. The detection of crack is generally based on the comparison of signal signatures of the fault and no-fault systems. The presence of sub-criticals in the CWT may be a best crack indicator but it is not always reliable. The addition of noise to the signal may sometimes lead to inaccurate results. So, there is a need to identify a parameter in addition to the sub-criticals. The phase angle between the two transverse vibrations or between the two signals (cracked and uncracked) can be a better crack indicator because it is very less sensitive to disturbance of the noise.

In the present work a new wavelet plot called cross wavelet transform (XWT) has been applied to the time signals to obtain the phase angles. The advantage of the XWT is that it clearly indicates the phase angles between all the frequency components in the signal (Grinsted et al 2004; Christopher \& Gilbert 1998). So, with the above XWT the phase angles between the sub-criticals of the two transverse signals and also the amplitudes of the sub-criticals are viewed in a single plot. Some parametric studies have also been carried out with crack depth, crack position along the rotor as variables, which can be used for diagnostics. The inverse problem of crack identification has been carried out using Artificial Neural Network (ANN). The vibration data (maximum value of CWT co-efficient) of the faulty system is fed to the ANN, once the ANN is trained then the crack parameters like position of crack, depth of crack can be inversely obtained from the ANN. 


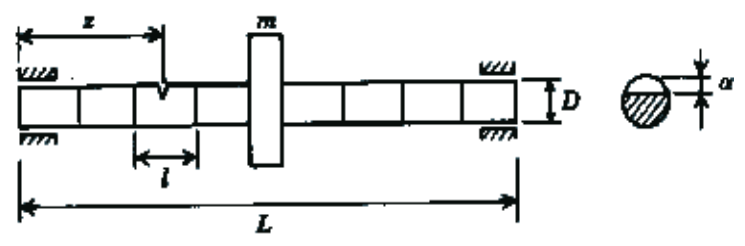

Figure 1. Finite element models of the rotor.

\section{Cracked rotor modelling}

The finite element model of a simple rotor-bearing system has been considered (Sekhar \& Prabhu 1994) as shown in (figure 1). The details of the cracked element used in the rotor shaft are given in (figure 2). Here ' $L$ ' is the total length of the shaft, ' $D$ ' the diameter, ' $l$ ' the element length, ' $m$ ' mass of the disc, ' $z$ ' location of the crack and ' $\alpha$ ' the crack depth.

The equation of motion of the complete rotor system in a fixed coordinate system can be written as

$$
[M]\{\ddot{q}\}+[D]\{\dot{q}\}+[K]\{q\}=\{F\} .
$$

The excitation matrix $\{F\}$ consists of the unbalance forces due to disc having mass $m$, eccentricity $e$ and the weight of the disc. The transverse breathing crack (Sekhar \& Prabhu 1994) has been considered in the present study. With the shearing action neglected and by using the strain energy, the flexibility coefficients for a bending element without a crack can be derived as in $C_{0}$.

$$
C_{0}=\left[\begin{array}{cccc}
l^{3} / 3 E I & 0 & 0 & l^{2} / 2 E I \\
0 & l^{3} / 3 E I & l^{2} / 2 E I & 0 \\
0 & l^{2} / 2 E I & l / E I & 0 \\
l^{2} / 2 E I & 0 & 0 & l / E I
\end{array}\right]
$$

Here $E I$ is the bending stiffness. The breathing action of the crack (Sekhar \& Prabhu 1994) i.e. it's opening and closing is illustrated in figure 3. During the shaft's rotation, the crack opens and closes, depending on the rotor deflection. For a large class of machines, the static deflection is much greater than the rotor vibration. With this assumption, the crack is closed when $\Phi=0$ and it is fully open when $\Phi=180$. The transverse surface crack on the shaft element introduces considerable local flexibility due to strain energy concentration in the vicinity of the crack tip under load. The additional strain energy due to the crack results in a

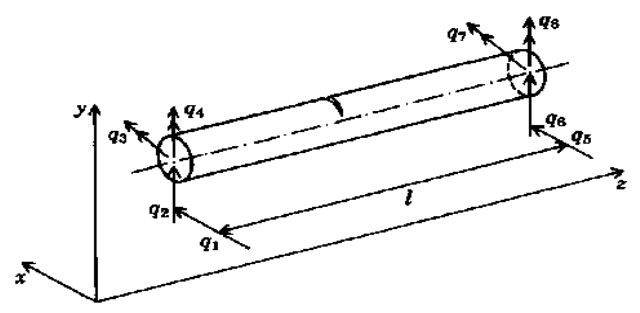

Figure 2. Cracked element. 


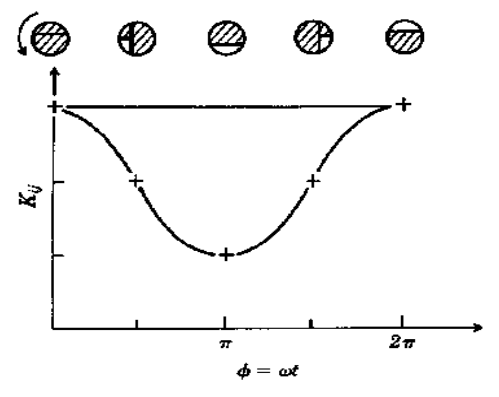

Figure 3. Breathing crack model.

local flexibility matrix $C_{c}$ which will be $C_{o p}$ and $C_{H C}$ for a fully open crack and half-open, half-closed conditions respectively

$$
\begin{gathered}
C_{o p}=\left(1 / F_{0}\right)\left[\begin{array}{cccc}
\bar{C}_{11} R & 0 & 0 & 0 \\
0 & \bar{C}_{22} R & 0 & 0 \\
0 & 0 & \bar{C}_{33} / R & \bar{C}_{43} / R \\
0 & 0 & \bar{C}_{43} / R & \bar{C}_{44} / R
\end{array}\right] \\
C_{H C}=\left(1 / 2 F_{0}\right)\left[\begin{array}{cccc}
\bar{C}_{22} R & 0 & 0 & 0 \\
0 & \bar{C}_{11} R & 0 & 0 \\
0 & 0 & \bar{C}_{44} / R & \bar{C}_{34} / R \\
0 & 0 & \bar{C}_{34} / R & \bar{C}_{33} / R
\end{array}\right] .
\end{gathered}
$$

Here $F_{0}=\pi E R^{2} /\left(1-v^{2}\right), R=D / 2$ and $v=0 \cdot 3$. The dimensionless compliance coefficients, $\bar{C}_{i j}$, are functions of non-dimensional crack depth $\bar{\alpha}$ (where $\bar{\alpha}=\alpha / D$ ). The total flexibility matrix for the cracked section is given as

$$
[C]=\left[C_{0}\right]+\left[C_{c}\right]
$$

The stiffness matrix of the cracked element ${ }^{4}$ is written as

$$
\left[K_{c}\right]=[T][C]^{-1}[T]^{T},
$$

where $[T]$ is the transformation matrix obtained from the equilibrium condition

$$
\left(q_{1}, q_{2}, \ldots, q_{8}\right)^{T}=[T]\left(q_{5}, \ldots, q_{8}\right)^{T} .
$$

When the shaft is cracked, during rotation the stiffness varies with time, or with angle. The variation may be expressed by a truncated cosine series

$$
[K]=\left[K_{0}\right]+\left[K_{1}\right] \cos \omega t+\left[K_{2}\right] \cos 2 \omega t+\left[K_{3}\right] \cos 3 \omega t+\left[K_{4}\right] \cos 4 \omega t
$$

Where $\left[K_{\eta}\right], \eta=0,1, \ldots, 4$ are the fitting coefficient matrices, determined from the known behaviour of the stiffness matrix at certain angular locations as explained in (Sekhar \& Prabhu 1994). 


\section{Results and discussions}

A steel shaft supported on two isotropic flexible bearings at both ends and having a disc at the centre, with the following data is considered for the analysis: shaft diameter $20 \mathrm{~mm}$, length $500 \mathrm{~mm}$; disc mass $5.5 \mathrm{~kg}$, polar moment of inertia $=0.01546 \mathrm{kgm}^{2}$, unbalance eccentricity $=0.01 \mathrm{~mm}$; bearing stiffness $=10^{5} \mathrm{~N} / \mathrm{m}$, damping $=100 \mathrm{Ns} / \mathrm{m}$. The acceleration applied to the rotor system to get the transient data is $30 \mathrm{rad} / \mathrm{s}^{2}$. The Houbolt time marching technique with a time step of $\Delta t=0.001 \mathrm{~s}$ has been used to model the system in the time domain. The Morlet mother wavelet has been chosen for all the CWT's, with a scale of 30 .

\subsection{Crack detection using wavelets}

The output of the transient analysis is the time signal as (shown in figure 4a), there is no significant difference or a unique feature between the un-cracked and cracked system. So, the time signal has to be processed to get the hidden features, those features can be used as crack indicators for diagnosis. The application of wavelet transform has proved to be better tool for crack detection (Prabhakar et al 2001; Sekhar 2003). The appearance of sub-critical peaks in the wavelet plots is a unique indication of crack in the system (Prabhakar et al 2001; Sekhar 2003). But till now only 2D CWT (shown in figure 4b) has been exploited to detect the crack. Even though the 2D CWT can effectively detect the crack through the sub-criticals as the crack indicators, it has two disadvantages.

(i) As shown in figure $4 \mathrm{~b}$, the 2D CWT can detect cracks only when the crack in the system is near and above 0.2 (crack depth/shaft diameter) which is a large crack.

(ii) It can only show the appearance of sub-critical peaks but the quantization of the same is not possible because it has only 2-axes (time vs. amplitude) as shown in figure $4 \mathrm{~b}$.

To overcome the above problems encountered in the 2D CWT's a new plot has been introduced which is the 3D CWT plot. The 3D plot clearly shows both time and frequency information along with the amplitude as shown in figure 4c. The data is of transient type so the inclined spectrum in figure $4 \mathrm{c}$ represents the increase in frequency along the time. The cup shape or cone shaped region in which the amplitudes are shown with different shades is called Cone of Influence (COI) (Grinsted et al 2004; Christopher \& Gilbert 1998). The CWT has edge artifacts because the wavelet is not completely localized in time. It is therefore useful to introduce a Cone of Influence (COI) in which edge effects can not be ignored. Here the COI is the area in which the wavelet power caused by a discontinuity at the edge has dropped to $e^{-2}$ of the value at the edge (Grinsted et al 2004). The data which is inside the COI has high significance and the data which is out side the COI has very low significance.

Figure 4 shows the comparison of time, 2D CWT and 3D CWT for different crack depths. As said earlier that crack cannot be detected using the time signal. The 2DCWT detects the crack clearly from 0.2 onwards, for small cracks like 0.1 or 0.12 it is very difficult to detect the crack from the 2DCWT as shown in figure $4 \mathrm{~b}$. The $3 \mathrm{DCWT}$ figure $4 \mathrm{c}$ is more effective because it starts detecting the cracks at its early stage of $0 \cdot 1$ and it clearly detects the $1 / 3^{\text {rd }}$ critical for a crack depth of $0 \cdot 12$ (as indicated by a pointer). In modern system like aero-engines which run at high speeds the cracks should be detected at its early stage so that the system can be prevented from catastrophic failure. So, the use of 3D CWT plots for diagnostics will be better compared to the 2D CWT plots. The effectiveness of the 3D CWT plots over 2D CWT plots is because better resolution is obtained from the 3D CWT's. As the 

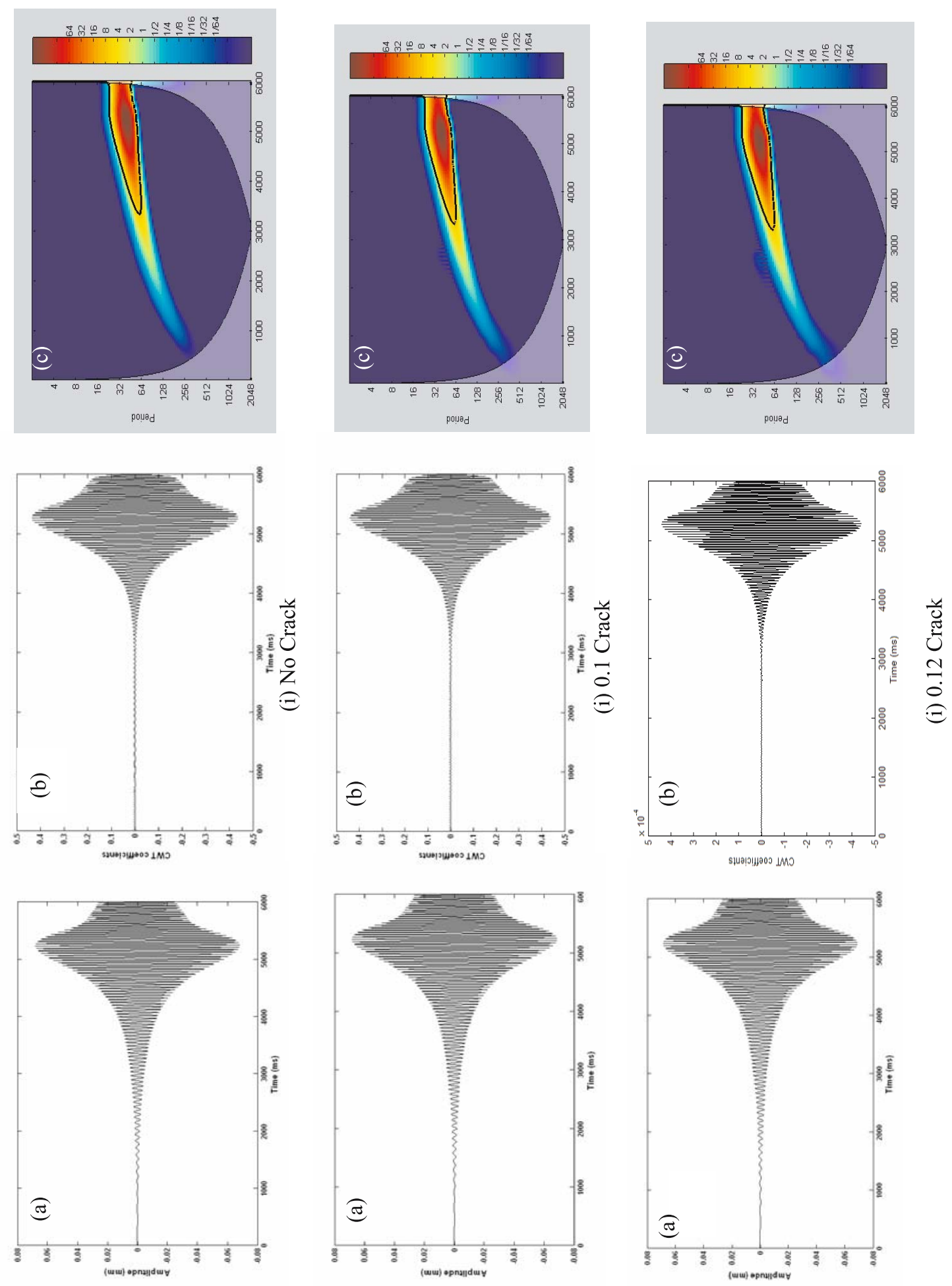

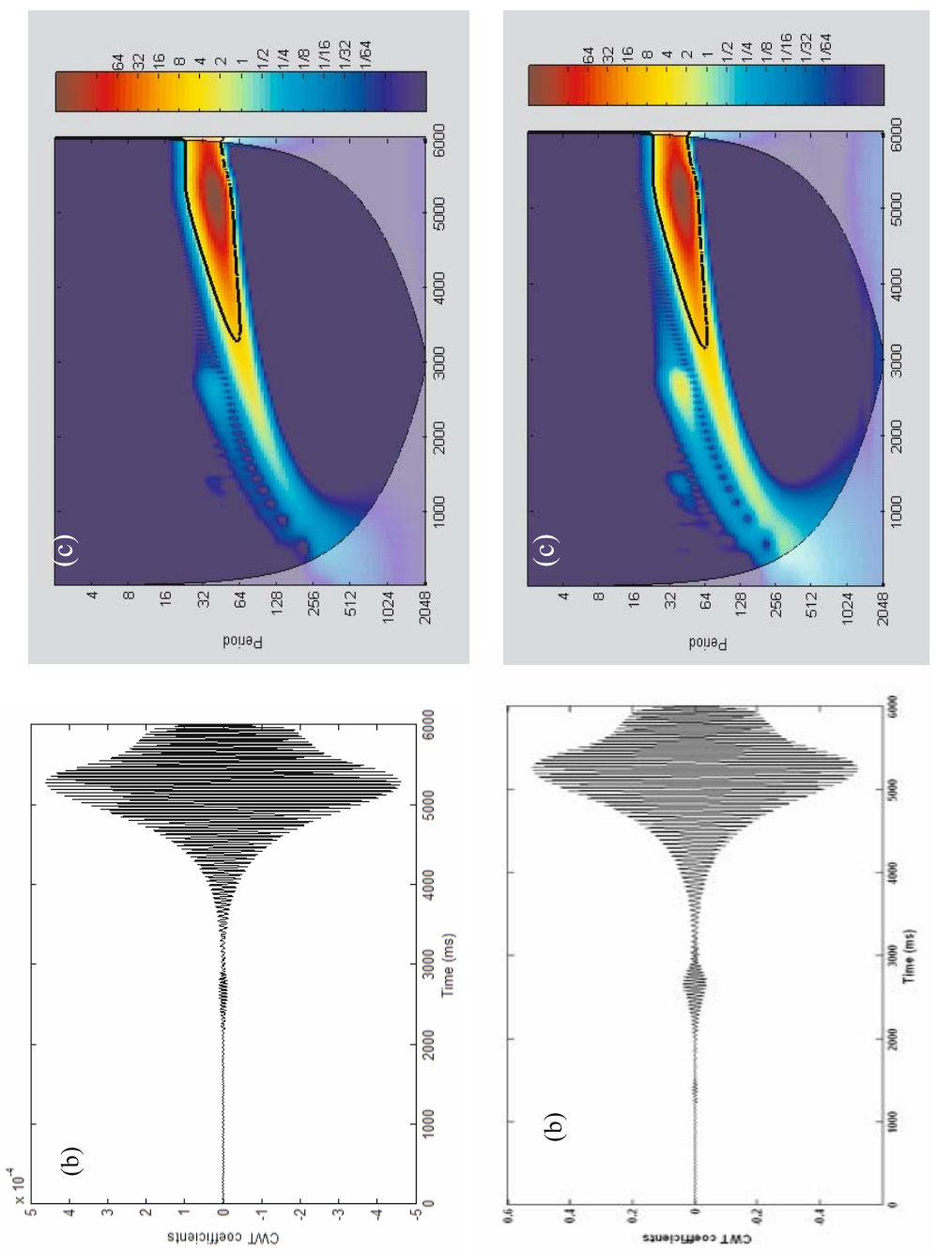

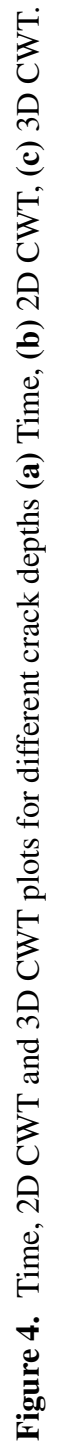
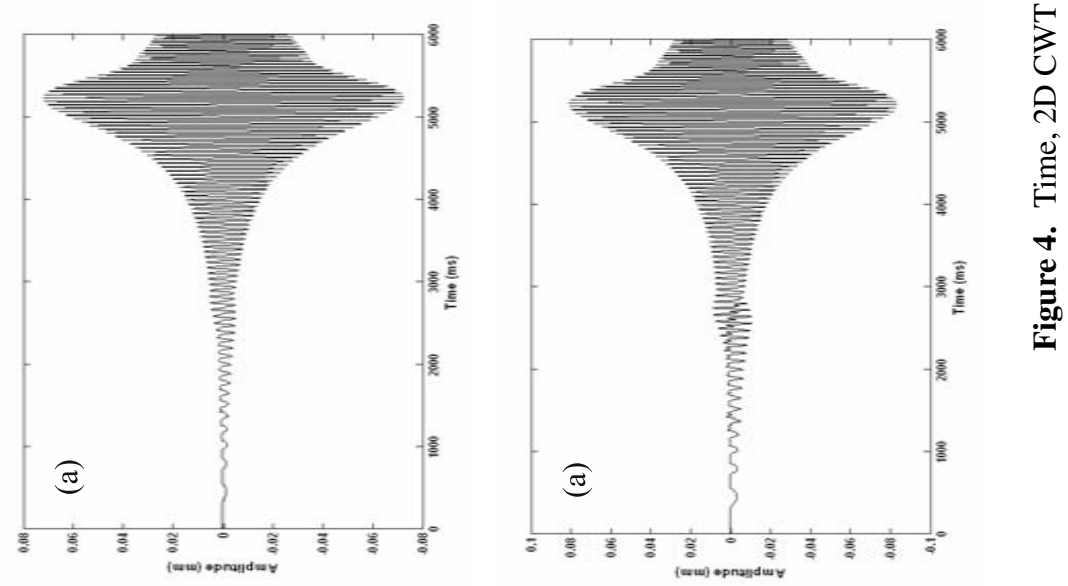
crack depth increases the split in sub-criticals can be clearly observed as shown in figure 4c. The splits in sub-criticals can be attributed to the crack propagation.

The presence of sub-criticals in the CWT may be a best crack indicator but it is not always reliable. The addition of noise to the signal may sometimes lead to inaccurate results. So, there is a need to identify a parameter in addition to the sub-criticals. The phase angle between the cracked and un-cracked signal can be a better crack indicator because it is very less sensitive to disturbance of the noise. In the present work, for the first time a new wavelet plot called cross wavelet transform (XWT) has been applied to the time signals to obtain the phase angles. As we are interested in the phase difference between the components of the two time series we need to estimate the mean and confidence interval of the phase difference. We use the circular mean of the phase over regions with higher than 5\% statistical significance that is outside the COI to quantify the phase relationship. This is a useful and general method for calculating the mean phase. The circular mean of a set of angles $\left(a_{i}, i=1 \ldots n\right)$ is defined as in (Grinsted et al 2004)

$$
a m=\arg (X, Y) \quad \text { with } X=\sum_{i=1}^{n} \cos \left(a_{i}\right) \quad \text { and } \quad Y=\sum_{i=1}^{n} \sin \left(a_{i}\right) .
$$

Figure 5 shows the XWT plots for different crack depths. In XWT plots the dark shaded region is the common power of the wavelet, the region marked with an approximate elliptical shape (figure 5(c and d)) is the sub-critical components of the two signals. The arrows in the plot represent the phase angles between the components in the two time signals. The arrows in the shaded portion or sub-critical zone have high significance compared to rest of the arrows. Figure 5e shows the XWT plot for no crack case, so the plot has a perfect match with zero phase angles (horizontal arrows indicate zero phase angle-zoomed view is shown in figure $5 \mathrm{e}$ ) between them. Figure $5 \mathrm{~b}$ is the XWT plot for a $0 \cdot 1$ crack case, there is no significance difference between the phase angles. Figure $5 \mathrm{c}$ is the XWT with $0 \cdot 2 \mathrm{crack}$, it is clearly observed (elliptical marked region-zoomed view is shown in figure $5 \mathrm{f}$ that there is phase difference between sub-critical components of the two signals. The same is clearly observed in the figure $5 \mathrm{~d}$ with $0 \cdot 3$ crack, but with higher phase magnitude.

In some cases there might be reasons for calculating the mean phase angle for each scale, and then the phase angle can be quantified to the crack depth. The advantage of the XWT is that it clearly indicates the phase angles between all the frequency components in the signal (Grinsted et al 2004). So, with XWT plot, the phase angles between the sub-criticals of the two signals (cracked and un-cracked) and also the amplitudes of the sub-criticals are viewed in a single plot. The XWT plot is an extended version of the CWT plot because the CWT has only amplitudes of vibration but the XWT has both amplitudes of vibration and the phase angle between the vibration components.

Figures 6, 7 and 8 show some parametric studies with crack depth, crack position along the rotor as variables. The sub-critical and critical peaks can be utilized for inverse tracking of the crack parameters and also for diagnostics purposes. It is clearly observed from the figure 6 that the maximum values of $1 / 3^{\text {rd }}$ critical peak is more significant and sensitive for crack detection, as the crack can be effectively diagnosed from its early stage of $20 \%$ (Crack depth*100/Shaft diameter). Figure 7 represents the maximum values of the $1 / 2$ critical peak, which is effective from $25 \%$ onwards. As shown from figure 8 , the maximum value of the main critical peak cannot be independently used for crack detection, because it is not a unique feature. So, either sub-critical data or sub-critical data along with main critical data can be used for crack diagnosis. 


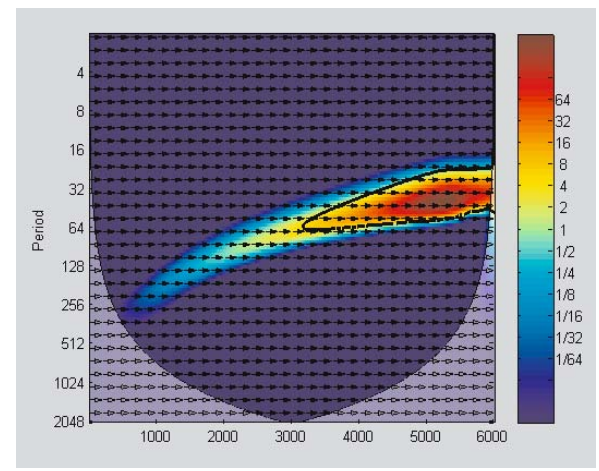

(a) No Crack

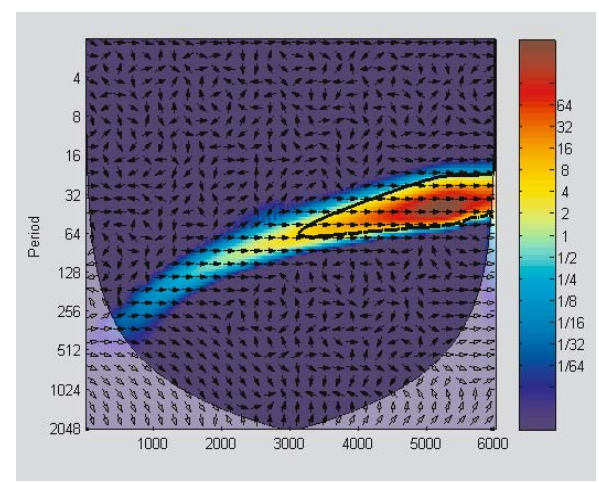

(c) $0.2 \mathrm{Crack}$

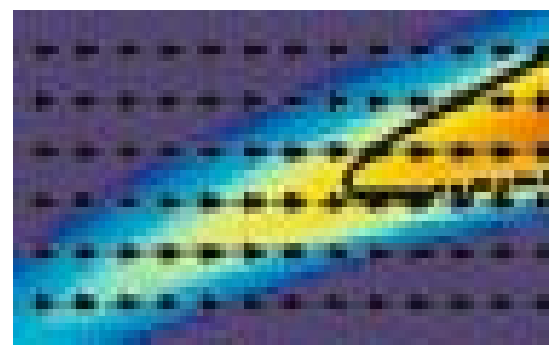

(e) The zoomed view of the enclosed portion of (a).

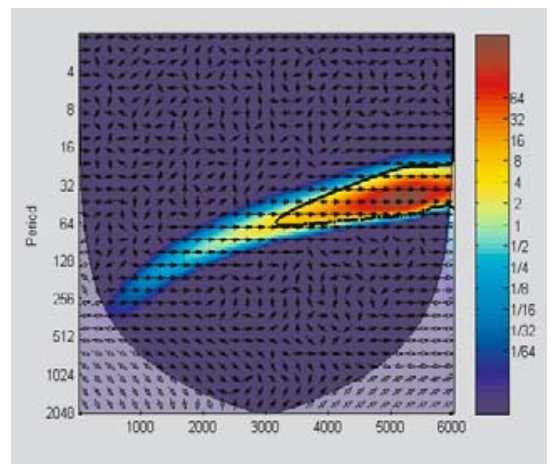

(b) 0.1 Crack

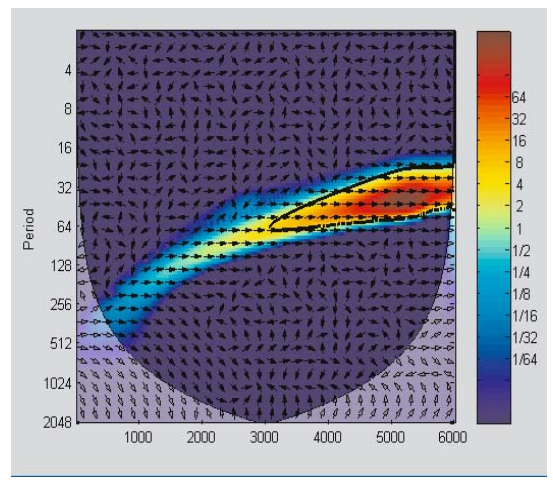

(d) 0.3 Crack

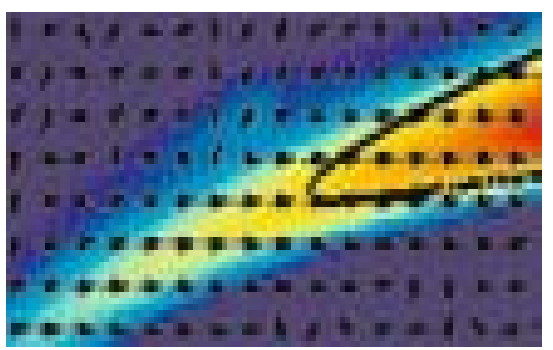

(f) The zoomed view of the enclosed portion of (c).

Figure 5. XWT plots for different crack depths.

\subsection{Determination of crack parameters using ANN}

Artificial neural networks provide a general, non-linear parameterized mapping between a set of inputs and a set of outputs. A network with three layers of weights and sigmoidal activation functions can approximate any smooth mapping and such a type has been used here. A typical supervised feed-forward multi-layer neural network, known as a BP (Back Propagation) neural network has been used in the present analysis (Zang \& Imregun 2001).

The network consists of three types of layers: the input layer ( 9 neurons -9 inputs) that receives the vibration data (CWT co-efficient), the hidden layer (12 neurons - trial and error) 


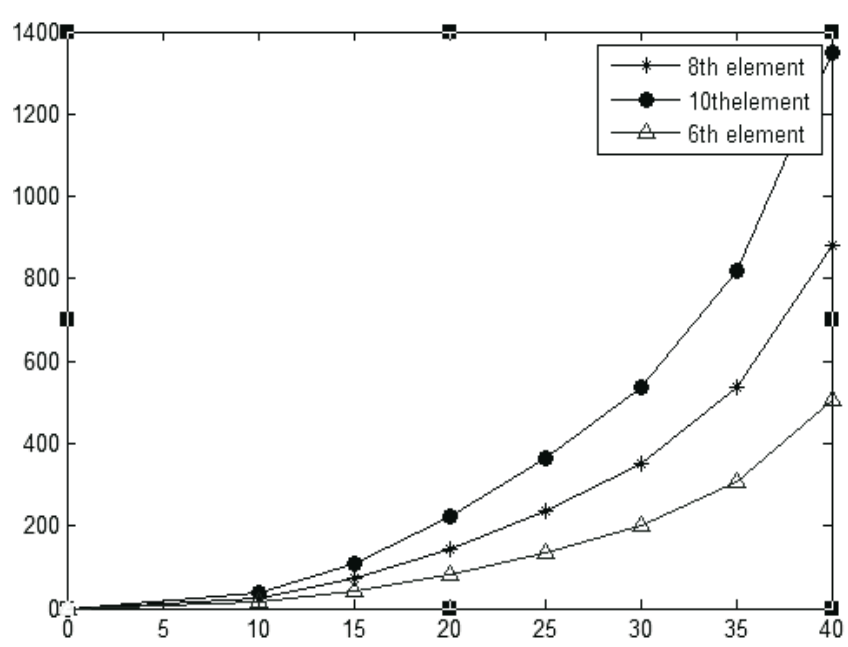

Figure 6. Amplitude of $1 / 3$ critical peak vs. crack depth.

which processes the data and the output layer ( 2 neurons -2 outputs) that provides the result of the analysis, i.e. depth of crack, position of crack. The training of a BP neural network is a two-step procedure. In the first stage, the network propagates input through each layer until an output is generated. The error between the actual output and the target output is then computed using mean square error (MSE). In the second stage, the calculated error is transmitted backwards from the output layer and the weights are adjusted accordingly in order to minimize the error. The training is stopped when the assumed value of the mean square error (MSE) is reached as shown in figure 9. The structural damage detection model is established through network training on known samples by the Levenberg-Marquardt learning algorithm (Matlab 7). The performance of a trained network can be measured to some extent by the

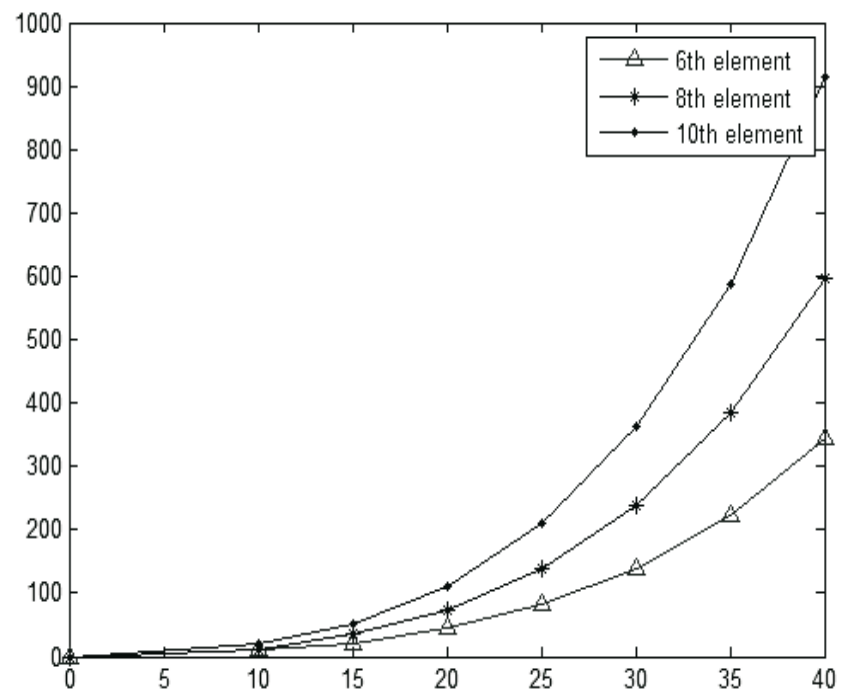

Figure 7. Amplitude of $1 / 2$ critical peak vs. crack depth. 


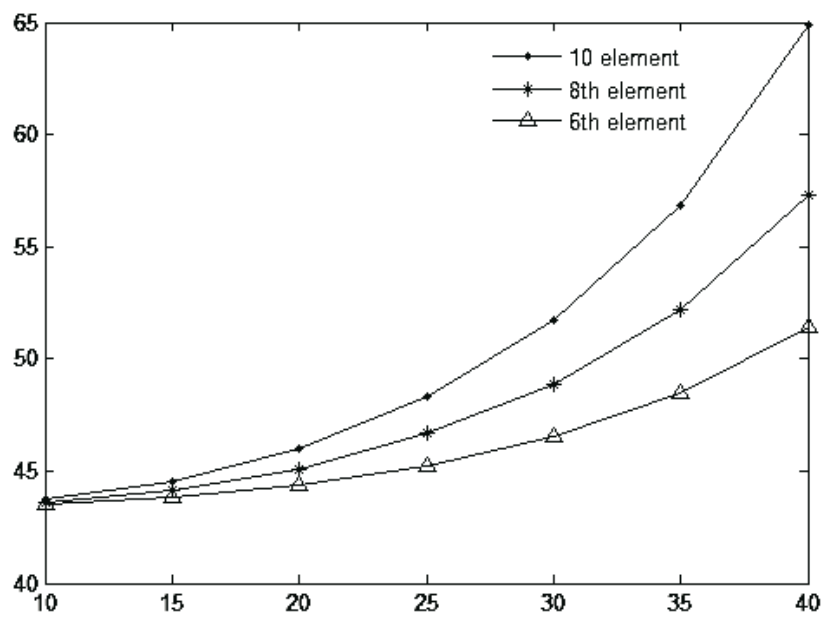

Figure 8. Amplitude of main critical peak vs. crack depth.

errors on the training, validation, and test sets, but it is often useful to investigate the network response in more detail. One option is to perform a regression analysis between the network response and the corresponding targets.

The network output and the corresponding targets are passed to regression analysis shown in figure 10. It returns three parameters. The first two correspond to the slope and the y-intercept of the best linear regression relating targets to network outputs. If there were a perfect fit (outputs exactly equal to targets), the slope would be 1 , and the y-intercept would be 0 . The third variable is the correlation coefficient (R-value) between the outputs and targets. It is a measure of how well the variation in the output is explained by the targets. If this number is equal to 1 , then there is perfect correlation between targets and outputs. The simulated data of the cracked system (generally called fault model) is passed to the ANN to train the Network.

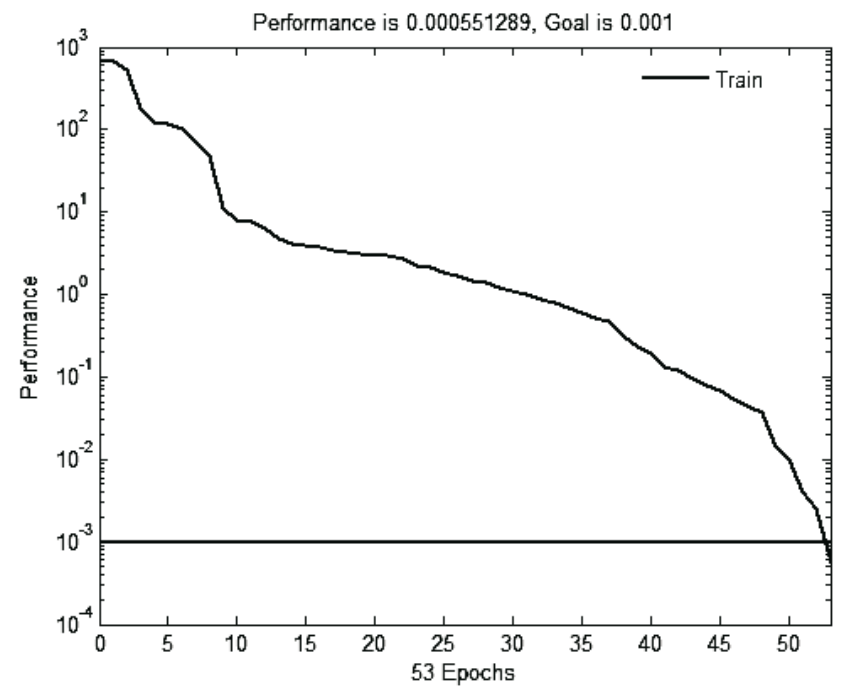

Figure 9. Training plot, MSE vs. Iterations (epochs). 


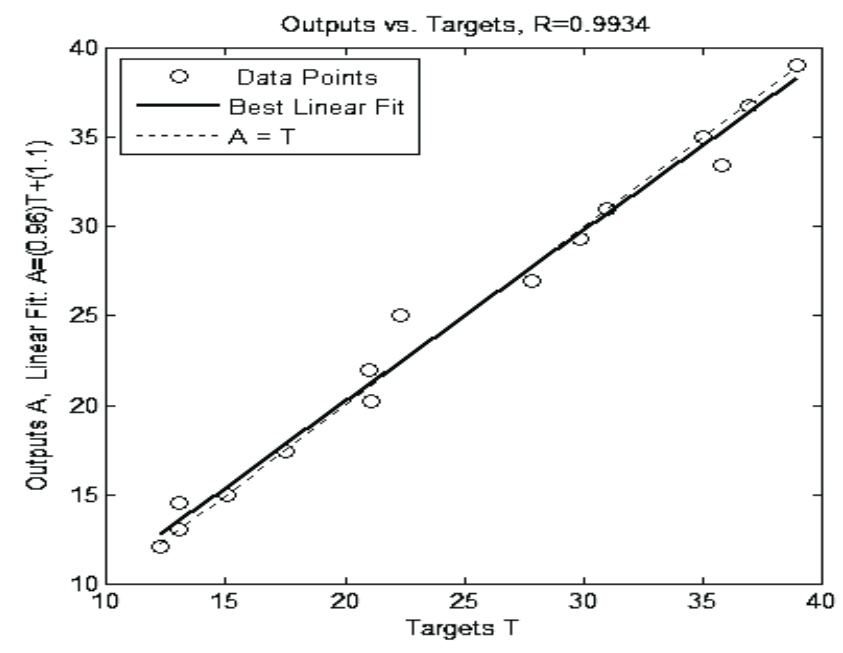

Figure 10. Regression of plot (Target value vs. ANN output).

Once the Network has been properly trained the test data is passed to obtain the depth of the crack, position of the crack. Figure 10 is the regression plot obtained for the test data of the system. The correlation coefficient (R-value) is 0.9934. The R-value clearly says that the present fit is a very good fit.

\section{Conclusions}

The transient analysis of rotor system with transverse breathing crack has been studied for flexural vibrations. The use of 3D CWT plots instead of 2D CWT plots is better, because the frequency components are both qualitatively and quantitatively indicated. The resolution of the 3D CWT plots is also better compared to the 2DCWT plots. Since, the sub-criticals are not always reliable to detect the crack. So, a new crack indicator has been identified using a relatively new signal processing technique called Cross Wavelet Transform (XWT). The XWT gives the phase angles of different frequency components along with the sub-critical peaks in a single plot. The advantage of the XWT has been exploited for the first time in rotor crack detection. The inverse problem of crack detection has been carried out using Artificial Neural Network (ANN).

\section{References}

Christopher Torrence, Gilbert P Compo 1998 A practical guide to wavelet analysis. Bull. Am. Meteorol. Soc. 79(1): 61-78

Dimarogonas A D 1996 Vibration of cracked structures: A state of the art review. Eng. Fracture Mech. 55: 831-857

Grinsted A, Moore J C, Jevrejeva S 2004 Application of the cross wavelet transform and wavelet coherence to geophysical time series

Matlab tool box, Version 7.0

Prabhakar S, Sekhar A S, Mohanty A R 2001 Detection and monitoring of cracks in a rotor-bearing system using wavelet transforms. Mechanical Systems and Signal Processing 15(2): 447-450 
Sabnavis G, Kirk R G, Kasarda M, Quinn D 2004 Cracked shaft detection and diagnostics: A literature review. The Shock and Vibration Digest 4: 287-296

Sekhar A S, Prabhu B S 1994 Transient analysis of a cracked rotor passing through the critical speed J. Sound and Vibration 173: 415-421

Sekhar A S 2003 Crack detection through wavelet transform for a run up rotor. J. Sound and Vibration 259(2): 461-472

Wauer J 1990 Dynamics of cracked rotors: a literature survey. Appl. Mech. Rev. 43(1): 13-17

Zang C, Imregun M 2001 Structural damage detection using artificial neural networks and measured FRF data reduced via principal component projection. J. Sound and vibration 242(5): 813-827 